\title{
CORRIGENDUM
}

\section{Aerobic denitrification in permeable Wadden Sea sediments}

Hang Gao, Frank Schreiber, Gavin Collins, Marlene M Jensen, Joel E Kostka, Gaute Lavik, Dirk de Beer, Huai-yang Zhou and Marcel MM Kuypers

The ISME Journal (2011) 5, 776; doi:10.1038/ismej.2010.166

Correction to: The ISME Journal (2010) 4, 417-426; doi:10.1038/ismej.2009.127

Since the publication of this paper, the authors have noticed an error in the list of co-authors and affiliations. The correct list of co-authors and affiliations is shown below.

Hang Gao ${ }^{1,2,3}$, Frank Schreiber ${ }^{1}$, Gavin Collins ${ }^{1,4}$, Marlene M Jensen ${ }^{1,5}$, Olivera Svitlica ${ }^{1}$, Joel E Kostka ${ }^{1,6}$, Gaute Lavik ${ }^{1}$, Dirk de Beer ${ }^{1}$, Huai-yang Zhou ${ }^{2,7}$ and Marcel MM Kuypers ${ }^{1}$

${ }^{1}$ Max Planck Institute for Marine Microbiology, Bremen, Germany; ' ${ }^{2}$ Guangzhou Institute of Geochemistry,
Chinese Academy Sciences, Guangzhou, PR China; ${ }^{3}$ Graduate School of Chinese Academy Sciences, Beijing, PR China; ${ }^{4}$ Current address: Department of Microbiology and Environmental Change Institute, National University of Ireland, University Road, Galway, Ireland; ${ }^{5}$ Current address: Institute of Biology and Nordic Center of Earth Evolution, University of Southern Denmark, Campusvej 55, DK-5230 Odense M, Denmark; ${ }^{6}$ Current address: Department of Oceanography, Florida State University, Leroy Collins Research Lab, Tallahassee, FL, USA and ${ }^{7}$ Current address: School of Ocean and Earth Science, Tongji University, Shanghai, PR China.

The authors would like to apologize for any inconvenience this may have caused. 